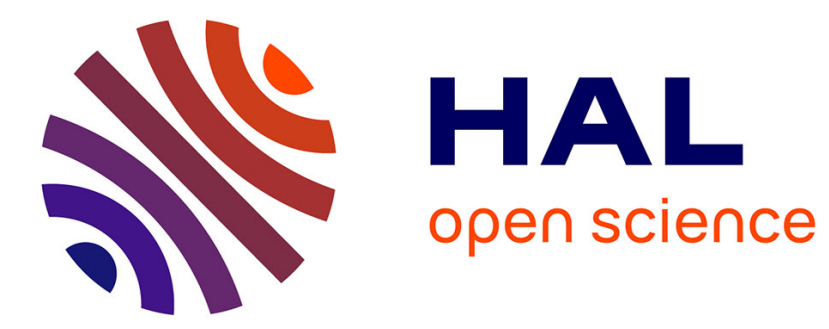

\title{
ÉNERGIE DE LIAISON D'ATOMES DE TRANSITION ADSORBÉS SUR LE TUNGSTÈNE
}

\author{
G. Allan, P. Lenglart
}

\section{To cite this version:}

G. Allan, P. Lenglart. ÉNERGIE DE LIAISON D'ATOMES DE TRANSITION ADSORBÉS SUR LE TUNGSTÈNE. Journal de Physique Colloques, 1970, 31 (C1), pp.C1-93-C1-98. 10.1051/jphyscol:1970116 . jpa-00213747

\section{HAL Id: jpa-00213747 https://hal.science/jpa-00213747}

Submitted on 1 Jan 1970

HAL is a multi-disciplinary open access archive for the deposit and dissemination of scientific research documents, whether they are published or not. The documents may come from teaching and research institutions in France or abroad, or from public or private research centers.
L'archive ouverte pluridisciplinaire HAL, est destinée au dépôt et à la diffusion de documents scientifiques de niveau recherche, publiés ou non, émanant des établissements d'enseignement et de recherche français ou étrangers, des laboratoires publics ou privés. 


\title{
ENERGIE DE LIAISON D'ATOMES DE TRANSITION ADSORBÉS SUR LE TUNGSTENE
}

\author{
G. ALLAN et P. LENGLART
}

Physique des Solides $\left({ }^{*}\right)$ I. S. E. N. 3, rue F.-Baes, 59, Lille

\begin{abstract}
Résumé. - On considère l'interaction en liaisons fortes d'une bande d'électrons « $s$ ) avec une surface (100). On admet que ce modèle décrit grossièrement la bande « $d$ ) du tungstène et on l'applique à l'étude de l'adsorption d'atomes de la $3^{\mathrm{e}}$ série de transition. Le potentiel d'interaction entre l'atome et le cristal, limité aux premiers voisins de l'atome adsorbé, est traité par la méthode des fonctions de Green compte tenu de la règle de Friedel.

Abstract. - The interaction between an $(s)$ band and a (100) surface is studied in the tight binding approximation. It is assumed that the model roughly describes the $(d)$ band of wolfram and it is applied to the binding adsorption of transition atoms of the third series. The interaction potential between the adatom and the crystal is limited to the nearest neighbours of the adatom and is described by the Green function method with Friedel's sum rule.
\end{abstract}

Introduction. - L'adsorption des métaux de la $3^{\mathrm{e}}$ série de transition sur le tungstène a été étudiée récemment par Plummer et Rhodin [1] par une méthode de désorption par effet de champ. Leurs résultats pour l'énergie d'adsorption doivent permettre une meilleure connaissance des liaisons en surface. Aucune étude théorique de l'adsorption des métaux de transition n'a été faite à ce jour : aussi nous essayons de traiter ce problème dans un modèle simplifié de surface [2]. Dans une première partie nous décrivons les approximations utilisées, méthode des liaisons fortes et structure cubique simple pour le tungstène et nous définissons de façon self-consistante l'effet de la surface (100) sur la structure électronique de $W$.

Dans la seconde partie nous introduisons une description self-consistante du problème de l'adsorption en montrant que la charge apportée par l'atome adsorbé est presque totalement écrantée sur la cellule d'adsorption, c'est-à-dire l'atome adsorbé et ses premiers voisins. On compare ensuite les résultats obtenus aux résultats expérimentaux et dans la conclusion, on met en évidence les résultats qui ne dépendent pas du modèle utilisé.

I. Surface (100) de W. - Des expériences récentes ont précisé la structure électronique du tungstène. En particulier la surface de Fermi a été observée par effet de Haas-van Alphen [3]. Nous retiendrons essentiellement que la bande $s$ de $W$ contient 1,28 électron

(*) Equipe de Recherche du C. N. R.S.

Cet article recouvre en partie le travail de thèse enregistré au C. N. R. S. sous le $\mathrm{n}^{\circ}$ A.0.3679 qui sera soutenu en 1970 par Guy Allan à la Faculté des Sciences d'Orsay pour l'obtention du grade de docteur d'état ès sciences physiques. par atome. Dans le problème de l'adsorption, le phénomène fondamental semble être l'interaction électronique entre un niveau atomique et la structure de bande du $W$. Afin d'aborder cette étude, nous utiliserons un modèle simplifié de description de la structure de bande et nous admettrons que la bande $d$ est à moitié pleine, ce qui ne peut entraîner une erreur fondamentale. De plus, nous décrirons cette bande $d$ dans l'approximation des liaisons fortes limitée aux premiers voisins en ne prenant qu'une orbitale par atome et en utilisant une structure cubique simple. Le caractère fondamental de la bande $d$, c'est-à-dire une forte densité d'états et une faible largeur est conservé en plaçant 10 électrons par atome dans le modèle de bandes obtenu. On prend $7 \mathrm{eV}$ pour largeur de bande de $W$, largeur raisonnable étant donné l'énergie de cohésion de ce métal $[8,9]$. On admet que la surface (100) est identique au plan (100) du cristal en volume, c'est-à-dire que l'on néglige la relaxation des atomes en surface.

Pour créer la surface, on coupe un cristal infini suivant un plan (100) (Fig. 1). L'effet de cette coupure sur la structure électronique pourra être représenté par un potentiel perturbateur que l'on traite par une méthode de déphasages self-consistante. En effet, la coupure introduit des variations de charges localisées au voisinage de la surface. On admettra que ces variations sont importantes seulement dans le plan de la surface et $n$ 'induisent de potentiel de self-consistance notable que dans ce plan. En utilisant les notations de la référence [2], le potentiel perturbateur $V$ entre fonctions de Wannier [4] localisées sur des plans parallèles à la surface est diagonal en $\mathbf{k}_{*}$, composante de $k$ dans 


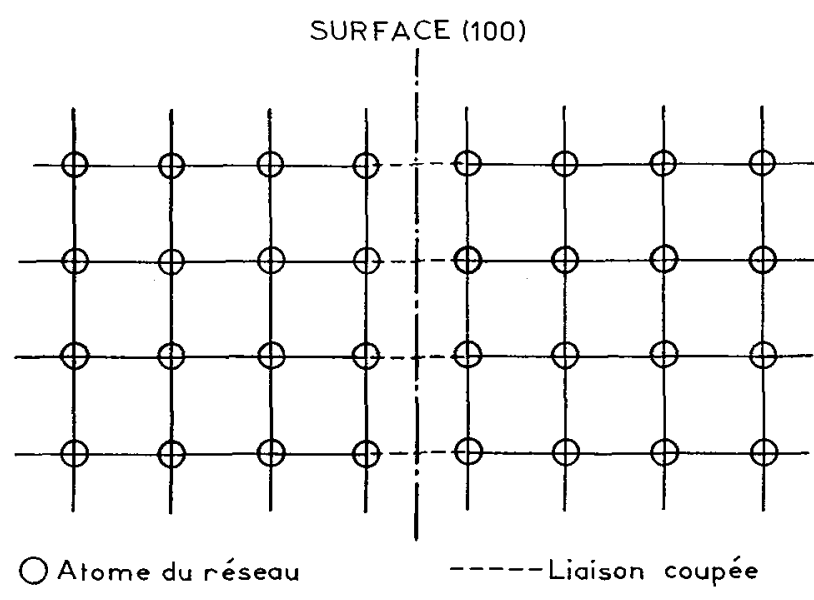

Fír. 1. - Modèle de surface.

le plan de la surface. $V\left(\mathbf{k}_{*}\right)$ ne comprend que quatre éléments de matrice entre les deux plans de la coupure

$$
V\left(\mathbf{k}_{*}\right)=\left(\begin{array}{cc}
\alpha^{\prime}+\delta V & \gamma \\
\gamma & \alpha^{\prime}+\delta V
\end{array}\right)
$$

où $\gamma$ est l'intégrale de résonance du cristal parfait,

$\alpha^{\prime}$ est égal à $1 / 6$ de l'intégrale de dérive, et $\delta V$ est l'élément de matrice intra-atomique du potentiel self-consistant.

Ce potentiel est déterminé en utilisant la règle de Friedel et en exprimant que la variation totale de charge au niveau de Fermi est nulle [6]. En effet, le fait d'avoir coupé les liaisons ne modifie pas le nombre total de charges. Dans le cas particulier utilisé, pour décrire $W$, bande $d$ à moitié pleine, le problème est symétrique entre électrons et trous, aussi le potentiel global self-consistant $\alpha^{\prime}+\delta V$ doit être nul, ce qui avait été trouvé en particulier dans la référence [2].

Le traitement de la surface (100) par la méthode des fonctions de Green permet de décrire la structure électronique du cristal coupé à partir de celle du cristal infini. En particulier, on démontre que, compte tenu des approximations choisies, les éléments de matrice des opérateurs de Green du cristal avec la surface dans la base des fonctions atomiques, satisfont à la relation :

$$
\begin{array}{ll}
G_{000}^{S}= & \left.\sum_{l=0}^{\infty}(-1)^{l}\left(\delta V+\alpha^{\prime}\right)^{l} G_{00_{l}}^{0}-G_{00_{l}+2}^{0}\right) \\
\text { où } \quad G_{000}^{S}=\left\langle 000\left|\frac{1}{E-H+i \varepsilon}\right| 000\right\rangle \\
G_{00 l}^{0}=\left\langle 000\left|\frac{1}{E-H+i \varepsilon}\right| 00 l\right\rangle
\end{array}
$$

sont les éléments de matrice des opérateurs de Green du cristal perturbé et du cristal infini, $\mid i j k>$ représentant l'orbitale atomique centrée au site

$$
\mathbf{R}=i \mathbf{a}_{x}+j \mathbf{a}_{y}+k \mathbf{a}_{z},
$$

l'origine étant choisie sur la surface.
II. Adsorption d'un atome de transition sur W. L'énergie de cohésion des métaux de transition et sa variation parabolique avec le remplissage de la bande $d$ est bien décrite en admettant que cette énergie est due aux seuls électrons $d$. De la même façon, il semble que l'énergie d'adsorption sera en majeure partie liée au réarrangement des électrons $d$ de l'atome libre dans la bande $d$ du tungstène. On admettra qu'un électron $s$ de l'atome libre s'étale dans la bande $s$ de $W$ avec un gain d'énergie sensiblement égal à l'énergie de promotion sd. Aussi dans ce modèle simplifié, la description de l'adsorption d'un atome dans la configuration $5 d^{n} 6 s^{2}$ sera traitée par l'interaction d'un niveau $5 d^{n+1}$ avec la bande $d$ de $W$. On admet que l'atome de transition $s^{\prime}$ adsorbe sur la surface en position cubique simple (Fig. 2). Entre l'atome adsorbé et le réseau,

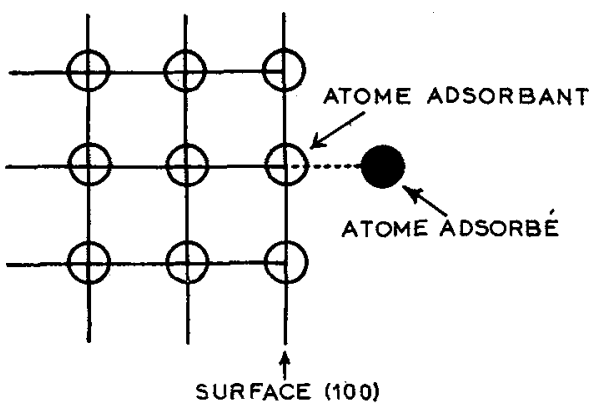

Fig. 2. - Cellule d'absorption.

l'interaction se crée par l'intermédiaire d'une intégrale de résonance et d'une intégrale de dérive que l'on choisit identiques à celles du réseau.

Soit $H_{S}$ et $H_{A}$ l'hamiltonien pour un électron $d$ d'un atome libre de $W$ et de l'atome libre quis'adsorbe

$$
\begin{gathered}
H_{S}\left|E_{0}>=E_{0}\right| E_{0}> \\
H_{A}\left|A>=E_{A}\right| A>
\end{gathered}
$$

le niveau $E_{A}$ est tiré des calculs de Herman et Skillma [10].

Nous prendrons $E_{0}$ comme origine des énergies et les notations suivantes

$$
\begin{aligned}
& \gamma=\left\langle R_{j}\left|V\left(\mathbf{r}-\mathbf{R}_{j}\right)\right| R_{j^{\prime}}>\right. \\
& \quad \text { avec } R_{j} R_{j^{\prime}} \text { premiers voisins } \\
& \left.\alpha=<R_{j}\left|V\left(\mathbf{r}-\mathbf{R}_{j^{\prime}}\right)\right| R_{j}\right\rangle
\end{aligned}
$$

où $V(\mathbf{r})$ est le potentiel de l'atome libre de $W$. Avec les approximations décrites précédemment, on traite le problème de l'adsorption par le formalisme de la diffusion. Formellement le problème est identique à l'étude d'une impureté de transition dans $W$.

- La charge supplémentaire apportée en surface est écrantée par les électrons $d$ du tungstène ; en effet la faible densité électronique des électrons $s$ rend leur rôle négligeable.

- L'écran à grande distance est total, aussi lapport de charges en surface ne peut modifier la densité 
électronique à grande distance de la surface, c'est-àdire qu'il n'y a pas de variation de niveau de Fermi.

- La self-consistance est alors obtenue en utilisant règle de Friedel [5] : la charge totale écrantée par les électrons de $W$ d'énergie inférieure à $E_{F}$ est égale à la charge apportée en surface.

- On admettra qu'au-delà du premier voisin de l'atome adsorbé, les variations de charges sont négligeables et $n$ 'induisent pas de potentiel de self-consistance.

Dans la base des orbitales atomiques le potentiel diffuseur $\mathcal{Y}$ qui s'étend alors sur l'atome adsorbé $A$ et l'atome du réseau premier voisin $R$ prend la forme

$$
\begin{array}{cc}
|R\rangle & |A\rangle \\
V=\langle R|\left(\begin{array}{ll}
-\alpha^{\prime}+\Delta V_{r} & -\gamma \\
-\gamma & -\alpha^{\prime}+\Delta V_{a}
\end{array}\right)
\end{array}
$$

où $\Delta V_{r}$ et $\Delta V_{a}$ sont les termes intra-atomiques des potentiels self-consistants provenant des variations locales de charges sur l'atome de réseau et sur l'atome adsorbé. En principe $\Delta V_{r}$ et $\Delta V_{a}$ peuvent être déterminés à partir des variations de charges $\Delta \rho_{r}$ et $\Delta \rho_{a}$. En fait un tel calcul nécessite la connaissance des termes de Coulomb et de corrélation, termes qu'il est délicat d'évaluer. Afin de traiter le problème nous envisagerons les deux modèles suivants :

modèle 1 : On néglige les termes de dérive dans la structure de bande de $W$ et on néglige le terme $\Delta V_{r}$ potentiel self-consistant sur l'atome du réseau $R$. La règle de Friedel détermine alors $\Delta V_{a}$ et on montre que la charge déplacée sur $R$ et $A$ est approximativement égale à la charge totale à écranter.

modèle 2 : Il semble alors que si l'on tient compte de $\Delta V_{r}$, l'écran sera amélioré. On admet donc que l'écran est total sur $R$ et $A$ :

$$
\Delta \rho_{a}+\Delta \rho_{r}=0 \text {. }
$$

Cette relation jointe à la règle de Friedel permet de traiter le problème de l'adsorption compte tenu ou non de la dérive.

II. 1. MoDÈLE 1. - Puisque le potentiel ne s'étend que sur $R$ et $A$, les seules fonctions de Green qui vont intervenir dans le problème de diffusion sont :

$$
\begin{aligned}
& G_{000}^{S}(E)=\left\langle R\left|\frac{1}{E-H+i \varepsilon}\right| R\right\rangle \\
& G^{A}(E)=\left\langle A\left|\frac{1}{E-H_{A}+i \varepsilon}\right| A\right\rangle .
\end{aligned}
$$

La partie utile de l'opérateur de Green prend la forme

$$
G(E)=\left(\begin{array}{cc}
G_{000}^{S}(E) & 0 \\
0 & G^{A}(E)
\end{array}\right)
$$

les termes non diagonaux étant nuls puisqu'il n'y a pas de liaison avant adsorption entre le réseau et l'atome $A$.
Le déphasage total $\eta(E)$ est donné par [7]

$$
\eta(E)=\operatorname{Arg} \operatorname{dét}(1-\vartheta G) \text {. }
$$

La charge totale écrantée par les électrons d'énergie inférieure à $E$ est :

$$
\Delta N(E)=-\frac{2 \times 5}{\pi} \eta(E)
$$

puisque l'on traite une bande $d$ en considérant une dégénérescence d'ordre 5 . La règle de Friedel

$$
\Delta N\left(E_{F}\right)=n+1=N_{a}
$$

détermine $\Delta V_{\boldsymbol{A}}$ :

$$
\begin{aligned}
\Delta V_{a}=E_{F}-E_{A}- & \gamma^{2} \operatorname{Re}\left[G_{000}^{S}\left(E_{F}\right)\right]- \\
& -\frac{\gamma^{2} \operatorname{Im}\left[G_{000}^{S}\left(E_{F}\right)\right]}{\operatorname{tg} \frac{N_{a} \pi}{10}}\left({ }^{*}\right) .
\end{aligned}
$$

On en déduit alors la variation de charges sur l'atome adsorbé :

$$
\begin{aligned}
& \Delta \rho_{a}=-\frac{10}{\pi} \times \\
& \quad \times \int^{E_{F}} \operatorname{Im}\left[\frac{G^{A}(E)}{1-\gamma^{2} G^{A}\left(E-\Delta V_{a}\right) G_{000}^{\mathrm{S}}(E)}\right] \mathrm{d} E
\end{aligned}
$$

et sur l'atome adsorbant

$$
\begin{aligned}
& \Delta \rho_{r}=-\frac{10}{\pi} \times \\
& \quad \times \int^{E_{F}} \operatorname{Im}\left[\frac{G_{000}^{S}(E)}{1-\gamma^{2} G^{A}\left(E-\Delta V_{a}\right) G_{000}^{S}(E)}\right] \mathrm{d} E .
\end{aligned}
$$

Les quantités $\Delta \rho_{a}, \Delta \rho_{r}$ et $\Delta \rho_{t}=\Delta \rho_{a}+\Delta \rho_{r}$ en fonction de $N_{a}$ sont données sur la figure 3. On voit que

- les variations de charges $\Delta \rho_{a}$ et $\Delta \rho_{r}$ sont de signes opposés et ne dépassent pas 0,5 électron ;

- la variation totale de charge $\Delta \rho_{t}$ ne dépasse jamais 0,15 électron. Il apparaît alors que la condition d'écran total est presque satisfaite.

Notons que dans ce modèle, si l'on introduit les termes de dérive on ne peut plus obtenir la self-consistance pour toute valeur de $N_{a}$, en particulier dans le cas de $N_{a}$ faible.

L'énergie de liaison $E_{\mathrm{L}}$ obtenue dans ce modèle est donnée par :

$$
\begin{aligned}
E_{\mathrm{L}}=\int^{E_{F}} E \Delta n(E) \mathrm{d} E & -N_{a} E_{A} \\
& -N_{a} \Delta V_{a}-\frac{1}{2} \Delta \rho_{a} \Delta V_{a}
\end{aligned}
$$

où $\Delta n(E)=\mathrm{d} / \mathrm{d} E(N(E))$ est la variation de densité d'états due à l'adsorption. Les termes

$$
N_{a} \Delta V_{a} \quad \text { et } \quad \frac{1}{2} \Delta \rho_{a} \Delta V_{a}
$$

(*) On désigne par $\operatorname{Re}(X)$ et $\operatorname{Im}(X)$ les parties réelles et imaginaires de $X$. 


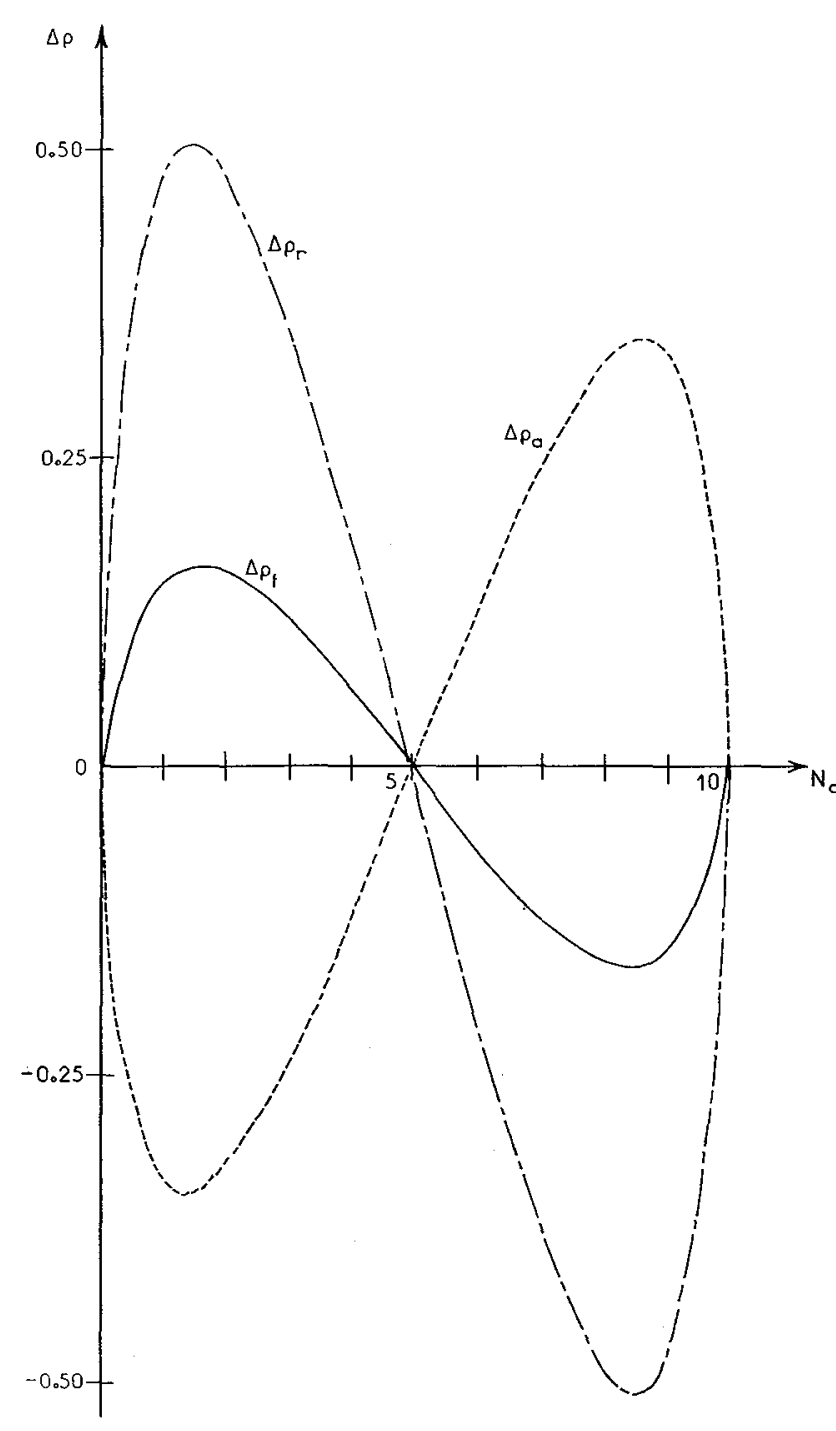

Fig. 3. - Variations de charge sur l'atome adsorbant $\Delta \rho_{a}$ sur l'atome adsorbé $\Delta \rho_{a}$ et sur la cellule d'absorption $\Delta \rho_{t}$.

tiennent compte de ce que l'on a compté deux fois les variations d'interactions coulombiennes en sommant les énergies à un électron [9]. La courbe donnant l'énergie de liaison en fonction de $N_{a}$ est indiquée sur la figure 4 ; elle sera discutée dans le paragraphe suivant.

Notons qu'il n'est pas correct de négliger $\Delta V_{r}$ puisque la variation de charge $\Delta \rho_{r}$ est plus importante que la variation $\Delta \rho_{a}$, aussi nous considérerons dans le modèle suivant un potentiel qui s'étend sur la cellule d'adsorption.

II.2. Modèle 2. - Dans ce modèle on traite le cas plus complet d'un potentiel self-consistant s'étendant sur les atomes $A$ et $R$.

On admet que l'écran est total sur $A$ et $R$ soit que

$$
\Delta \rho_{r}+\Delta \rho_{a}=0
$$

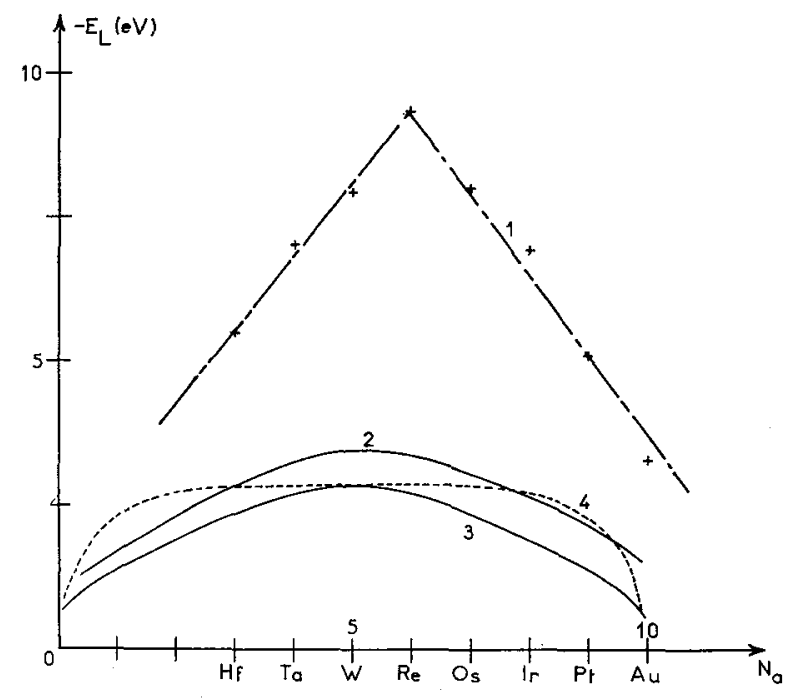

FIG. 4. - Energies de liaison des atomes de transition de $3^{\mathrm{e}}$ série sur $W .1)$ Courbe expérimentale [1] ;2) Ecran total avec dérive ; 3) Ecran total sans dérive ; 4) $\Delta V_{r}=0$.

Cette équation jointe à la règle de Friedel (16) permet de déterminer $\Delta \rho_{R}$ et $\Delta \rho_{A}$. On obtient alors

$$
\begin{gathered}
\Delta \rho_{r}=-\frac{10}{\pi} \int^{E_{F}} \operatorname{Im} \times \\
\times\left[\frac{G_{000}^{S}(E)}{1-\Delta V_{r}} \overline{G_{000}^{S}(E)-\gamma^{2} G^{A}\left(E-\Delta V_{a}\right) G_{000}^{S}(E)}\right] \mathrm{d} E
\end{gathered}
$$

et

$$
\Delta \rho_{a}=-\frac{10}{\pi} \int^{E_{F}} \operatorname{Im} \times
$$$$
\times\left[\frac{G^{A}\left(E-V_{a}\right)\left(1-\Delta V_{r} G_{000}^{S}(E)\right.}{1-\Delta V_{r} G_{000}^{S}(E)-\gamma^{2} G^{A}\left(E-\Delta V_{a}\right) G_{000}^{S}(E)}\right] \mathrm{d} E
$$

où

$$
\begin{gathered}
\Delta V_{a}=E_{F}-E_{A}- \\
-\frac{\gamma^{2} \operatorname{Im}\left[G_{000}^{S}\left(E_{F}\right)\right]+\gamma^{2} \operatorname{tg}\left(\frac{N_{a} \pi}{10}\right) R\left[G_{000}^{S}\left(E_{F}\right)\right]}{\operatorname{tg}\left(\frac{N_{a} \pi}{10}\right)\left(1-\Delta V_{R} R\left[G_{000}^{S}\left(E_{F}\right)\right]\right)-} \\
-\Delta V_{R} \operatorname{Im}\left[G_{000}^{S}\left(E_{F}\right)\right]
\end{gathered}
$$

et $\Delta V_{R}$ est déterminé numériquement.

Les quantités sont représentées sur la figure 5. On remarque que :

- Les variations de charges $\Delta \rho_{A}$ et $\Delta \rho_{R}$ restent faibles quelle que soit la charge de l'atome adsorbé. 

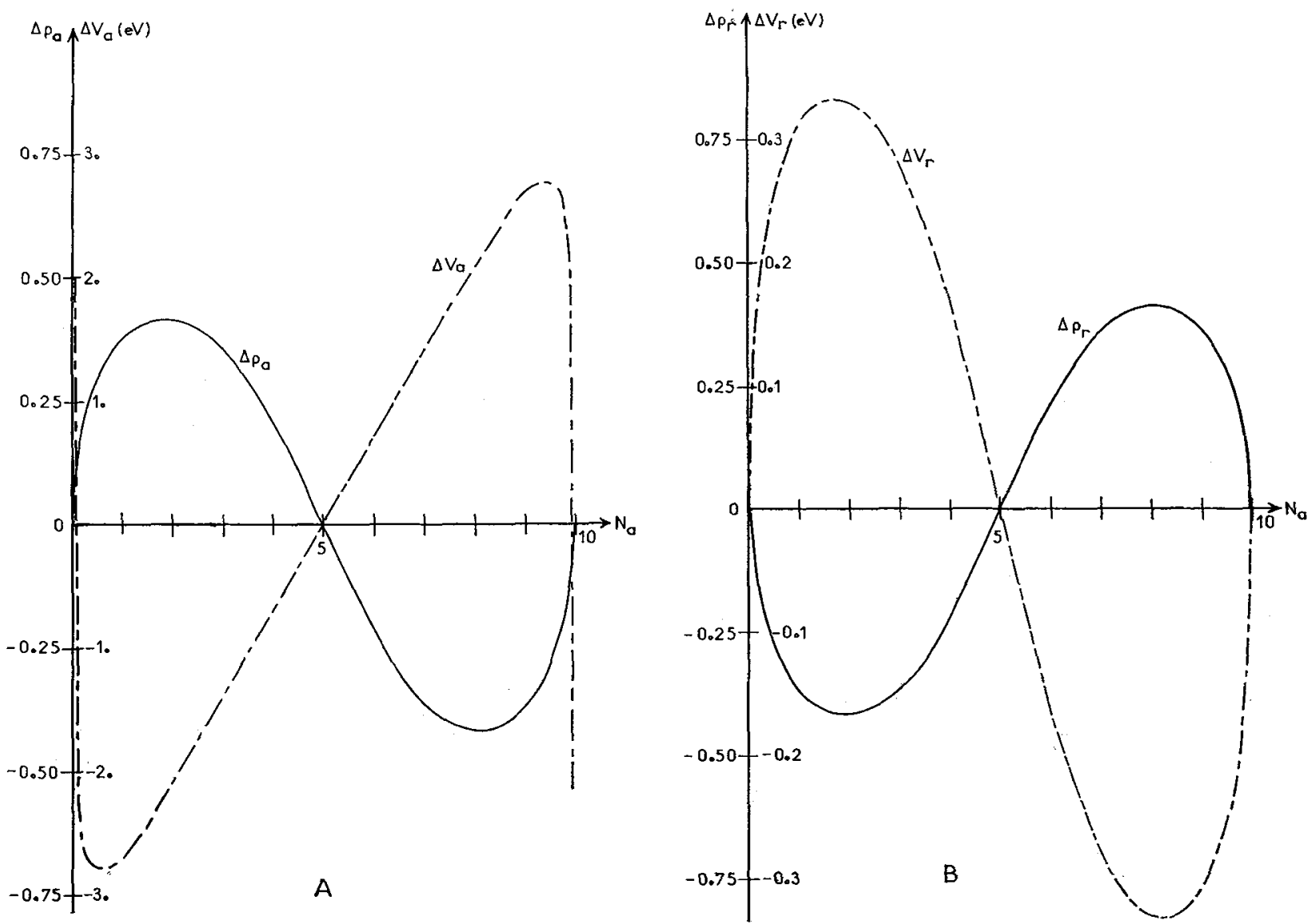

FIG. 5. - A) Variations de charge et de potentiel sur l'atome adsorbé;

$B$ ) Variations de charge et de potentiel sur l'atome adsorbant.

- Dans un large voisinage de $N_{A}=5$, les variations de potentiel sont proportionnelles aux variations de charges soit :

$$
\begin{aligned}
& \Delta V_{R}=-J_{r} \Delta \rho_{r} \\
& \Delta V_{A}=-J_{a} \Delta \rho_{a}
\end{aligned}
$$

avec $J_{r}=0,77 \mathrm{eV}$ et $J_{a}=3,13 \mathrm{eV}$.

Dans ce cas l'énergie de liaison devient :

$$
\begin{aligned}
E_{L}=+\int^{E_{F}} E \Delta n(E) \mathrm{d} E-N_{a} E_{a}-N_{a} \Delta V_{a}- \\
-5 \Delta V_{r}-\frac{1}{2} \Delta \rho_{r} \Delta V_{r}-\frac{1}{2} \Delta \rho_{a} \Delta V_{a} .
\end{aligned}
$$

L'énergie de liaison obtenue est représentée sur la figure 4 ainsi que les résultats expérimentaux.

On y remarque en particulier deux choses :

a) L'énergie de liaison obtenue est toujours nettement plus faible que l'énergie expérimentale. Cela tient essentiellement au fait que dans ce modèle de structure simple, il n'y a qu'une seule liaison entre atome adsorbé et le cristal.

b) Les courbes d'énergie de liaisons théoriques avec un ou deux potentiels self-consistants sont symétriques par rapport à la valeur $N_{a}=5$, contrairement aux résultats expérimentaux. Il semble que cette dissymétrie, que l'on retrouve dans le cas de l'énergie de cohé- sion et l'énergie de formation d'une lacune [9], aussi dans le cas de la tension superficielle [6], soit due à l'intégrale de dérive.

On peut tenir compte formellement de la dérive dans ce problème d'adsorption en admettant que les potentiels self-consistants calculés précédemment se composent d'une partie due à la dérive, soit :

$$
\begin{aligned}
& \Delta V_{a}=\alpha^{\prime}+\Delta V_{a}^{\prime} \\
& \Delta V_{r}=\alpha^{\prime}+\Delta V_{r}^{\prime} .
\end{aligned}
$$

On en déduit immédiatement la nouvelle forme de l'énergie de liaison $E_{e}^{\prime}$

$$
E_{\mathrm{L}}^{\prime}=E_{\mathrm{L}}-\left(N_{a}+5\right) \alpha^{\prime} .
$$

L'énergie de liaison obtenue possède alors la bonne dissymétrie (Fig. 4). Cette dissymétrie reste néanmoins faible puisque l'on a seulement dans ce modèle un seul terme de dérive.

Conclusion. - Nous avons essayé, sur un modèle simple, de décrire l'adsorption des métaux de transition en considérant le cas du tungstène. La méthode peut s'appliquer à tout autre cas de métal de transition adsorbant. Certains résultats obtenus semblent 
devoir se conserver dans un modèle plus élaboré : c'est en particulier le cas de

- l'écran presque total sur la cellule d'adsorption, c'est-à-dire atome adsorbé et ses premiers voisins ;

- la dissymétrie de la courbe d'énergie de liaisons dans le cas de $W$ reliée à l'intégrale de dérive ;

- la variation de l'énergie de liaison avec $N_{a}$.

Par ailleurs il semble que la valeur de l'énergie de liaison trouvée, nettement plus faible que la valeur expérimentale soit liée au fait que nous n'avons pris qu'une seule liaison entre l'atome adsorbé et le cristal. Dans le cas réel de $W$, l'atome adsorbé en position cubique centré posséde quatre liaisons ce qui entraîne certainement une énergie de liaison nettement plus grande.
L'énergie d'adsorption d'un atome de transition sur un métal de transition semble bien liée principalement aux électrons $d$ du métal qui forment la majeure partie de l'écran au potentiel en surface apporté par l'atome adsorbé. On a ici un problème très voisin de celui d'une impureté de transition dans une matrice de transition avec la difficulté supplémentaire apportée par la surface. Pour obtenir une description plus correcte de l'adsorption, il faudrait bien sûr traiter d'abord une structure de métal plus réaliste, mais aussi avoir une meilleure connaissance théorique des liaisons en surface.

Remerciements. - Nous tenons à remercier Monsieur le Professeur J. Friedel pour ses nombreux conseils et le docteur G. Léman pour nos stimulantes discussions.

\section{Bibliographie}

[1] Plummer (E. W.) and Rhodin (T. N.), J. Chem. Phys., 1968, 49, 3479.

[2] Allan (G.) and Lenglart (P.), Surf. Science, 1969, 15, 101 .

[3] Girvan (R. F.), Gold (A. V.) and Phimirs (R. A.), J. Phys. Chem. Solids, 1968, 29, 1485.

[4] Brown (R. A.), Phys. Rev., 1967, 156, 889.

[5] Friedel (J.), Nuovo Cimento Suppl., 1958, 7, 287.
[6] Allan (G.), à paraître.

[7] Toulouse (G.), Solid State Comm., 1966, 4, 593.

[8] Friedel. (J.), The Physics of Metals, ed. J. Ziman, Cambridge University Press, 1969, p. 363.

[9] LANNoo (M.) and AlluAN (G.), à paraître.

[10] Herman (F.) and Skillman (S.), Atomic structure Calculations Prentice-Hall, Inc., 1963. 